\title{
Froin's syndrome secondary to disseminated staphylococcus infection in a COVID-19 patient.
}

\author{
Adrian Soto Mota ${ }^{1}$ \\ ${ }^{1}$ Instituto Nacional de Ciencias Medicas y Nutricion Salvador Zubiran
}

June 3, 2021

\begin{abstract}
Bacterial CNS are rare in COVID 19 patients. We describe a patient with severe pneumonia and meningitis that developed Froin's syndrome by Staphylococcus aureus; this suggests that immune dysregulation in patients with COVID-19 facilitate disseminating commensal bacterial infections from the skin or the mucous membranes. Keywords: Meningitis, Froin's syndrome, COVID-19.
\end{abstract}

Main title: Froin's syndrome secondary to disseminated staphylococcus infection in a COVID-19 patient. Adrian Soto Mota.

Metabolic Diseases Research Unit.

Daniel Rebolledo García and Guillermo García Ramos.

Neurology and Psychiatry Department.

Instituto Nacional de Ciencias Médicas y Nutrición "Salvador Zubirán"

Vasco de Quiroga 15, Colonia Belisario Dominguez, Delegación Tlalpan. CP 14080.

México City, México.

\begin{abstract}
Bacterial CNS are rare in COVID 19 patients. We describe a patient with severe pneumonia and meningitis that developed Froin's syndrome by Staphylococcus aureus; this suggests that immune dysregulation in patients with COVID-19 facilitate disseminating commensal bacterial infections from the skin or the mucous membranes.
\end{abstract}

Keywords: Meningitis, Froin's syndrome, COVID-19.

\section{Key clinical message}

Patients with severe COVID 19 increase the risk for bacterial meningitis.

In the absence of systemic confounders, late awakening in patients with COVID-19

under orotracheal intubation should be candidates for a lumbar puncture.

\section{Clinical presentation}

A 56-year-old man with a history of diabetes mellitus since 2015 presented with headache, fever, and cough in August 2020. Over the next two days, his respiratory symptoms progressed and finally, presented pneumonia. He refrained from seeking hospital care and self-medicated with acetaminophen. Four days after the onset 
of his respiratory symptoms, he presented confusion and decreased alertness; thus, he was taken to our hospital's emergency department.

At admission, he was drowsy (Glasgow score $=13$ points (Eyes: 3, Verbal: 4, Motor: 6 ) and no signs of meningeal irritation were observed. He presented involuntary movements in the limbs, with predominance in hands and feet. These movements were arrhythmic, intermittent, and paroxysmal suggestive of segmental myoclonus. The rest of the neurological examination was not substantial. The physical examination only revealed a laceration of the fourth and fifth toes of the right foot with purulent material, highly suspicious of cellulitis.

A brain CT scan with contrast and perfusion maps was unremarkable and a thoracic CT scan found a groundglass pattern, disseminated in both lungs, compatible with severe SARS CoV 2 pneumonia. Intravenous dexamethasone $6 \mathrm{mg}$ qd and high requirements of supplementary oxygen were initiated, and COVID-19 was confirmed with RT-PCR.

Results of serum test were resumed in table 1. The rest of the protocol was negative.

Due to the high suspicion of central nervous system infection, a lumbar puncture was performed. It yielded a cloudy xanthochromic cerebrospinal fluid (CSF) with elevated protein $(429 \mathrm{mg} / \mathrm{dl}$ ), glucose $117 \mathrm{mg} / \mathrm{dl}$, white blood cells (WBC) 296 cells/ $\mu$ l (272 neutrophils, 24 lymphocytes, 478 crenocytes), and a visible clot.

Peripheral blood, urine and lung expectoration samples for culture were obtained and empirical antibiotic therapy was initiated with meningeal doses of ceftriaxone and vancomycin. Additionally, the surgery team washed the wound in the left foot, and obtained biopsy samples for culture. The pathological examination was consistent with osteomyelitis.

Thus, the cardiology team made a transesophageal echocardiogram which did not document changes compatible with endocarditis. Instrumented ophthalmologic examination was negative for retinal embolism.

Cerebrospinal fluid culture, blood cultures, lung expectoration, and foot wound culture were positive for multidrug-sensitive S. aureus. Therefore, antibiotic therapy was continued with ceftriaxone and rifampicin.

After two days of treatment, our patient's alert status improved but, he was still disoriented. However, his respiratory symptoms progressed, and he developed a right pleural effusion which was successfully drained. A control serum test showed decreased leukocytes (11.4 x 103), neutrophilia (86.6\%), lymphopenia (6.6\%), decreased hemoglobin $(7.1 \mathrm{~g} / \mathrm{dL})$ and a severe elevation in D-dimer $(237,296 \mathrm{ng} / \mathrm{ml})$ were found. After this, the infectiology team recommended to escalate his antibiotic therapy to meropenem and linezolid.

Since his consciousness deteriorated again, a new sample of CSF was obtained. It yielded a cloudy, xan-

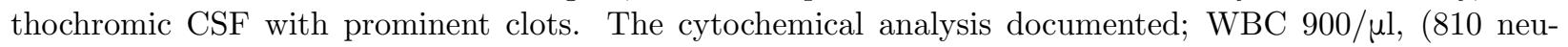
trophils, 90 lymphocytes); glucose $86 \mathrm{mg} / \mathrm{dl}$ and an even more elevated protein concentration $(808.1 \mathrm{mg} / \mathrm{dl})$ that the previously sample. It was impossible to obtain a third sample of CSF because of its prominent clotting which occluded the needle after a few drops of CSF (Figure 1).

Staphylococcus aureus persisted in all three lumbar puncture samples. RT-PCR for SARS-CoV-2 in the CSF was negative. Despite the adequate antibiotic treatment, the patient presented multiple organ failure and died twelve days after admission.

\section{Discussion}

To our knowledge, this case is the first report of Froin syndrome in a patient with COVID19. The available reports of cases with neurological symptoms and COVID-19 have been highly heterogeneous and Froin's phenomenon, syndrome, or pseudo-syndrome was not reported in any of them.

There is no CSF protein concentration cutoff for developing Froin's syndrome. This syndrome was initially described in patients with solid neoplasms and chronic meningitis due to tuberculosis or cryptococcus and vasculitis have been described as well. 
Recently, Neumann et al. described the cerebrospinal fluid findings in patients $(n=33)$ with neurological disease and COVID19. CSF analysis showed pleocytosis, increased protein (100-1200 mg/L), and lactate (1$3 \mathrm{mmol} / \mathrm{L}$ ). There was variability in the results of RT-PCR for SARS-CoV-2, with most tests on CSF being negative. Inflammatory CSF in patients with COVID-19 and neurological involvement does not necessarily correlate with severity or with an additional meningeal infectious process.

Rhea's recent work demonstrated in murine models the entry of the SARS CoV 2 virus into the neurons via the Spike (S1) protein, with sequestration of S1 in endothelial cells and glycocalyx affection. Disruption of the blood-brain barrier by endothelitis may indirectly contribute to the local inflammatory process, interleukin production, and synthesis of specific intrathecal antibodies, which may also explain some cases of immunemediated neuropathies, such as Guillain Barré syndrome.

A recent case series $(\mathrm{n}=42)$ by Cusumano et al. found $1.4 \%$ of $\mathrm{S}$. aureus bacteriemia in patients with severe COVID-19 which increased their mortality at 14 (54.8\%) and 30 days (66.7\%) after hospital admission. Predisposing factors were comorbidities diabetes mellitus, arterial hypertension, mechanical ventilatory support, and placement of invasive vascular access devices. Another study found a $16 \%$ prevalence of superinfections in critically ill COVID-19 patients $(\mathrm{n}=621)$. The infectious sites reported were pneumonia $(95 \%)$, abscesses or empyema (3.5\%), and septic emboli (1.5\%). Pathogens includedAcinetobacter baumannii, Staphylococcus aureus, Pseudomonas aeruginosa, and Klebsiella pneumoniae. However, there are no cohorts of central nervous system infections of bacterial origin in patients with COVID-19 described in the literature.

This case suggests that immune dysregulation in patients with COVID-19 could facilitate disseminating commensal bacterial infections from the skin or the mucous membranes and could increase the morbidity and mortality in these cases.

\section{Conflict of interest}

The authors declare that they have no conflict of interest.

\section{References}

1. Garispe A, Naji H, Dong F, Arabian S, Neeki M. Froin's Syndrome Secondary to Traumatic and Infectious Etiology. Cureus. 2019 Dec 6;11(12):e6313. doi: 10.7759/cureus.6313 .

2. Neumann B, Schmidbauer ML, Dimitriadis K, Otto S, Knier B, Niesen WD, Hosp JA, Günther A, Lindemann S, Nagy G, Steinberg T, Linker RA, Hemmer B, Bösel J; PANDEMIC and the IGNITE study groups. Cerebrospinal fluid findings in COVID-19 patients with neurological symptoms. J Neurol Sci. 2020 Nov 15;418:117090. doi: 10.1016/j.jns.2020.117090 .

3. Rhea, E.M., Logsdon, A.F., Hansen, K.M. et al. The S1 protein of SARS-CoV-2 crosses the blood-brain barrier in mice. Nat Neurosci 24, 368-378 (2021).https://doi.org/10.1038/s41593-020-00771-8.

4. Jaclyn A Cusumano, Amy C Dupper, Yesha Malik, Elizabeth M Gavioli, Jaspreet Banga, Ana Berbel Caban, Devika Nadkarni, Ajay Obla, Chirag V Vasa, Dana Mazo, Deena R Altman, Staphylococcus aureus Bacteremia in Patients Infected With COVID-19: A Case Series, Open Forum Infectious Diseases, Volume 7, Issue 11, November 2020, ofaa518, https://doi.org/10.1093/ofid/ofaa518

5. Clancy CJ, Schwartz IS, Kula B, Nguyen MH. Bacterial Superinfections Among Persons With Coronavirus Disease 2019: A Comprehensive Review of Data From Postmortem Studies. Open Forum Infect Dis. 2021 Feb 4;8(3):ofab065. doi: 10.1093/ofid/ofab065.

\section{Hosted file}

Figure 1..docx available at https://authorea.com/users/417724/articles/524730-froin-ssyndrome-secondary-to-disseminated-staphylococcus-infection-in-a-covid-19-patient

\section{Hosted file}


Table 1 Froin syndrome.docx available at https://authorea.com/users/417724/articles/524730froin-s-syndrome-secondary-to-disseminated-staphylococcus-infection-in-a-covid-19patient 